\title{
Replication and Meta-analysis of the Association between BDNF Val66Met Polymorphism and Cognitive Impairment in Patients Receiving Chemotherapy
}

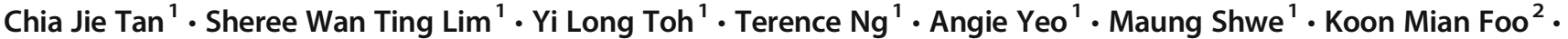 \\ Pat $\mathrm{Chu}^{3}$ - Amit Jain ${ }^{4}$ - Si-Lin $\mathrm{Koo}^{4}$ - Rebecca A. Dent ${ }^{4}$ - Raymond Chee Hui $\mathrm{Ng}^{4}$ - Yoon Sim Yap ${ }^{4}$ Elaine H. Lim ${ }^{4}$. \\ Kiley Wei-Jen Loh ${ }^{4}$. Wen Yee Chay ${ }^{4}$. Guek Eng Lee ${ }^{4}$. Tira Jing Ying Tan ${ }^{4}$. Sok Yuen Beh ${ }^{4} \cdot$ Mabel Wong $^{4}$. \\ Jack Junjie Chan ${ }^{4}$. Chiea Chuen Khor ${ }^{5,6}$ - Han Kiat Ho ${ }^{1}$ - Alexandre Chan ${ }^{1,7,8}$
}

Received: 21 September 2018 / Accepted: 24 October 2018 / Published online: 31 October 2018

(C) The Author(s) 2018

\begin{abstract}
Cancer-related cognitive impairment (CRCI) adversely affects cancer patients. We had previously demonstrated that the $B D N F$ Val66Met genetic polymorphism is associated with lower odds of subjective CRCI in the multitasking and verbal ability domains among breast cancer patients receiving chemotherapy. To further assess our previous findings, we evaluated the association of BDNF Val66Met polymorphism with subjective and objective CRCI in a temporally separate cohort of patients and pooled findings from both the original $(n=145)$ and current $(n=193)$ cohorts in a meta-analysis. Subjective CRCI was assessed using FACT-Cog. Objective CRCI was evaluated using computerized neuropsychological tests. Genotyping was carried out using Sanger sequencing. The association of BDNF Val66Met genotypes and CRCI was examined with logistic regression. A fixed-effect meta-analysis was conducted using the inverse variance method. In the meta-analysis $(n=338)$, significantly lower odds of CRCI were associated with Met allele carriers based on the global FACT-Cog score $(\mathrm{OR}=0.52$, 95\% CI 0.29-0.94). Furthermore, Met allele carriers were at lower odds of developing impairment in the domains of memory $(\mathrm{OR}=0.34,95 \% \mathrm{CI}: 0.17-0.70)$, multitasking $(\mathrm{OR}=0.33,95 \% \mathrm{CI}$ : $0.18-0.59)$, and verbal ability $(\mathrm{OR}=0.46,95 \% \mathrm{CI}: 0.24-0.88)$. Consistent with the previous study, lower odds of subjective CRCI among patients with the $B D N F$ Met allele was observed after adjusting for potential confounders in the multitasking $(\mathrm{OR}=0.30,95 \% \mathrm{CI}: 0.14-0.67)$ domain. In conclusion, carriers of the $B D N F$ Met allele were protected against global subjective CRCI, particularly in the domains of memory, multitasking, and verbal ability. Our findings further contribute to the understanding of CRCI pathophysiology.
\end{abstract}

Keywords Cancer-related cognitive impairment $\cdot$ BDNF $\cdot$ Genetic polymorphism $\cdot$ Breast cancer $\cdot$ Chemotherapy

Electronic supplementary material The online version of this article (https://doi.org/10.1007/s12035-018-1410-4) contains supplementary material, which is available to authorized users.

Alexandre Chan

phaac@nus.edu.sg

1 Department of Pharmacy, Faculty of Science, National University of Singapore, Block S4, 18 Science Drive 4, Singapore 117543, Singapore

2 Department of Pharmacy, K.K. Women's and Children's Hospital, Singapore, Singapore

3 Singapore Cord Blood Bank, Singapore, Singapore
4 Division of Medical Oncology, National Cancer Centre, Singapore, Singapore

5 Human Genetics, Genome Institute of Singapore, Singapore, Singapore

6 Glaucoma Research Group, Singapore Eye Research Institute, Singapore, Singapore

7 Department of Pharmacy, National Cancer Centre, Singapore, Singapore

8 Duke-NUS Graduate Medical School, Singapore, Singapore 


\section{Introduction}

Commonly known in literature as "chemobrain" or "chemofog," subtle yet notable alterations in cognitive function are often observed in breast cancer patients receiving chemotherapy [1]. Manifesting as both patient-reported subjective complaints and objective changes detected by neuropsychological tests, cancer-related cognitive impairment (CRCI) has been reported to include memory loss, concentration deficit, and the decreased ability to multitask [2]. Evidence has shown that CRCI negatively affects the quality of life of cancer patients and the ability to cope with demands in their daily lives [3]. As its etiology is not yet fully understood, CRCI remains a subject of significant research. Ongoing work has suggested possible factors that may influence the risk of CRCI, such as pro-inflammatory cytokines, psychosocial determinants including anxiety and fatigue, and numerous genetic markers [4-6]. Among candidate genes that have been investigated are COMT, $A P O E$, and $B D N F[6,7]$.

The $B D N F$ gene expresses brain-derived neurotrophic factor (BDNF), which is a neurotrophic factor vital for neuronal survival, growth, and neural circuit maintenance [8]. The $B D N F$ Val66Met single nucleotide polymorphism (SNP), which leads to substitution of valine with methionine at codon 66 , is a functional polymorphism widely studied in neurological conditions such as schizophrenia and Parkinson's disease $[9,10]$. Our research group has discovered that the $B D N F$ Val66Met polymorphism is associated with a lower risk of developing self-perceived CRCI in breast cancer patients [7], where carriers of the Met allele had lower odds of reporting subjective CRCI in the cognitive domains of verbal ability $(\mathrm{OR}=0.34,95 \% \mathrm{CI}=0.12-0.90)$ and multitasking $(\mathrm{OR}=$ $0.37,95 \% \mathrm{CI}=0.15-0.91)$. However, other studies have suggested that carrying the Met allele may be associated with poorer perseveration, verbal memory abilities, and task switching [11]. Therefore, whether carriers of the Met allele are truly protected against cognitive decline remains controversial, implying that the $B D N F$ Val66Met polymorphism may contribute to varying cognitive function [11].

As false positives are commonly observed in genetic association studies [12], further replication attempts are required to confirm associations that were initially observed, in order to provide stronger evidence on the impact of genetic determinants on CRCI. A deeper understanding of these genetic factors will allow the identification of cancer patients at a higher risk of CRCI for potential interventions. Therefore, in this study, we aim to evaluate the association of $B D N F$ Val66Met polymorphism with subjective and objective CRCI in a temporally separate cohort of patients and pool findings from both the original and current cohorts in a meta-analysis.

\section{Methods}

\section{Study Design}

This was a multicenter, prospective cohort study conducted at three ambulatory cancer centers between February 2014 and December 2017 in Singapore. This study was approved by SingHealth Institutional Review Board (CIRB2014/754/B) and written informed consent was obtained from all patients.

\section{Study Population}

Eligible participants must fulfill the following inclusion criteria: (i) at least 21 years old, (ii) diagnosed with stages I to III breast cancer, (iii) scheduled to receive chemotherapy, (iv) has no prior history of chemotherapy and/or radiotherapy, (v) able to read and understand either English or Mandarin, and (vi) has Eastern Cooperative Oncology Group (ECOG) performance status score of 0 or 1 .

Patients were excluded from the study if they were (i) incapable of providing verbal/written consent or (ii) diagnosed with neuropsychiatric disorders and/or brain metastasis that might result in poor cognitive function.

\section{Study Procedures}

Upon recruitment, demographic data and clinical information of participants were collected via patient interviews and from electronic medical records. Participants were prospectively evaluated at three time points: before start of chemotherapy (T1), 6 weeks after start of chemotherapy (T2), and 12 weeks after start of chemotherapy (T3). At each time point, participants completed assessments of both subjective and objective CRCI. In addition, healthrelated quality of life, anxiety, and fatigue were assessed using selfadministered questionnaires. English and Chinese versions of each study tool were available. All assessments took approximately $45 \mathrm{~min}$ to complete and were conducted by trained interviewers.

\section{Assessment of Subjective Cognitive Impairment}

The Functional Assessment of Cancer Therapy-Cognitive Function (FACT-Cog) version 3 was used to evaluate patients' self-perceived CRCI within the past 7 days [13]. FACT-Cog comprises 37 items in 6 domains of cognitive disturbances, which are mental acuity, concentration, memory, verbal ability, functional interference, and multi-tasking ability. Each item is evaluated on a 5-point Likert scale. Both English and Chinese versions of FACT-Cog have been validated and demonstrated satisfactory psychometric properties [14].

Subjective CRCI is defined as a reduction of at least 10.6 points in the FACT-Cog total score at T2 or T3 compared to baseline based on a previously determined minimal clinically 
important difference (MCID). Decline in a particular cognitive domain is defined as a reduction of at least $15 \%$ from a participant's baseline score at T2 or T3 [15].

\section{Assessment of Objective Cognitive Impairment}

Objective cognitive assessment was carried out using the Cambridge Neuropsychological Test Automated Battery (CANTAB), a language-independent neuropsychological testing research software. In this study, the CANTAB test battery contained five tests: reaction time (RTI), paired associates learning (PAL), spatial working memory (SWM), attention switching task (AST), and rapid visual information processing (RVP) that assessed response speed, learning and memory, working memory, multitasking, and sustained attention, respectively, yielding a total of nine measures. The direction of one measure, $A^{\prime}$, was reversed so higher scores indicate poorer cognitive performance for all measures. These tests have been validated and have shown sensitivity to capturing alterations in neuropsychological performance [16-18].

Reliable change indices (RCI) were computed to reflect cognitive changes in participants. RCI were obtained by subtracting CANTAB scores at T2 or T3 from baseline scores, adjusting for practice effects and dividing by the standard error of difference. Practice effects and standard error of difference were estimated from a control population using similar testing intervals. Objective cognitive decline is defined as an RCI of less than -2 at either $\mathrm{T} 2$ or T3.

\section{Assessment of Fatigue}

Fatigue was evaluated with the Brief Fatigue Inventory (BFI) [19]. BFI measures the severity of fatigue and the impact of fatigue on daily functioning in the past $24 \mathrm{~h}$ on a numerical scale of 0 to 10 . Six aspects of daily functioning were assessed: general activity, mood, walking ability, normal work, relations with other people, and enjoyment of life. A higher score indicates greater level of fatigue.

\section{Assessment of Anxiety}

The Beck Anxiety Inventory (BAI) was employed to measure anxiety in participants [20,21]. BAI is a validated questionnaire consisting of 21 items describing subjective, somatic, or panic-related symptoms of anxiety on a scale of 0 to 3 . A higher total score indicates greater level of anxiety.

\section{Assessment of Insomnia}

The European Organization for Research and Treatment of Cancer Quality of Life Questionnaire (EORTC QLQ-C30) assesses health-related quality of life (HRQoL). In this study, we focused on the single-item scale rating insomnia, which is measured on a 4-point Likert scale. A higher score indicates increased severity of insomnia. Both English and Chinese versions of QLQ-C30 have been validated for use in cancer patients in Singapore [22, 23].

\section{Genotyping}

At baseline, a 10-ml blood sample was collected from participants in an ethylene diamine tetraacetic acid (EDTA) tube and centrifuged at $2500 \mathrm{rpm}$ for $10 \mathrm{~min}$ within $40 \mathrm{~min}$ of collection. The buffy coat was extracted and at stored at $-80{ }^{\circ} \mathrm{C}$ until analysis.

Using QIAamp DNA Blood Mini Kit (QIAGEN), genomic DNA from the buffy coat was isolated. The region with the $B D N F$ Val66Met polymorphism was amplified via polymerase chain reaction (PCR) using the following specific and optimized primers: 5'-GGACTCTGGAGAGC GTGAA-3' (forward) and 5'-CGTGTACAAGTCTG CGTCCT-3' (reverse). Genotyping of the PCR products was subsequently conducted by AITbiotech employing automated Sanger sequencing with a 3730xl DNA Analyzer (Applied Biosystems). AITbiotech was blinded to clinical outcomes of participants. To ensure quality control, genotyping was done for both the forward and reverse DNA strands.

\section{Statistical Analysis}

All statistical analyses were conducted with STATA Version 15 (StataCorp 2017). Descriptive statistics were used to summarize demographics and clinical characteristics of participants. Deviation of genotypes from Hardy-Weinberg equilibrium was assessed using chi-squared test with one degree of freedom. Evaluation of the associations between the $B D N F$ Val66Met polymorphism and CRCI was done using logistic regression assuming a dominant model. Potential confounders age, race, menopausal status, chemotherapy regimens, years of education, and additionally for subjective CRCI, anxiety, depression, and insomnia were adjusted for [24, 25]. Sensitivity analyses was performed assuming a general genetic model with each genotype classified as a distinct class. To examine the relationship between anxiety and fatigue with BDNF genotype, linear mixed-effect models were employed with the presence of Met allele and time incorporated as fixed effects and intercepts varied as a random effect by each subject. To combine findings from both the original and current cohort, adjusted odds ratios from both studies were pooled in a fixed-effect meta-analysis using the inverse variance method. All statistical tests were two-sided, and $p$ values less than 0.05 were considered statistically significant. 


\section{Sample Size Calculation}

Sample size calculation was performed using Quanto 1.2.4. In our original study, statistically significant association of CRCI with $B D N F$ genotype was observed for the cognitive domains of verbal ability $(\mathrm{OR}=0.34)$ and multitasking $(\mathrm{OR}=0.37)$ [7]. The latter, which yielded a smaller effect size, was used for sample size estimation in this study. Based on an expected allelic frequency of 0.5 in a dominant model and predicted prevalence of impairment at 0.3 [7], a total of 167 participants is required to yield statistical power of $80 \%$ and type 1 error of $5 \%$. Anticipating an attrition rate of $20 \%$, a final sample size of 209 was targeted.

\section{Results}

\section{Patient Characteristics}

A total of 209 patients were recruited. However, 15 participants withdrew from the study ( 2 patients refused chemotherapy and 13 declined to complete study procedures) and 1 patient did not provide blood samples for genotyping. Therefore, 193 participants were included in the final analysis (Fig. 1). The demographic characteristics of patients who dropped out and those who remained in the study did not differ significantly. The mean $( \pm \mathrm{SD})$ age of participants was $51.9 \pm 8.9$ years old. Majority of the participants were of Chinese ethnicity $(79.8 \%)$ and had at least high school education $(84.5 \%)$. More than half received radiotherapy $(66.3 \%)$, underwent mastectomy (63.2\%), and completed anthracycline-based chemotherapy (64.8\%). Demographic and clinical characteristics of participants in both the current and original cohorts are comparable (Table 1).

\section{Genotype and Allele Frequencies}

All participants included in the final analysis were successfully genotyped for the $B D N F$ Val66Met polymorphism. Val/Val and Met/Met homozygous genotypes accounted for $26.9 \%$ and $20.7 \%$ of the observed genotypes, respectively, while the remaining $52.3 \%$ comprised the heterozygous genotype. Val and Met allele frequencies were approximately equivalent. No deviation from Hardy-Weinberg equilibrium was detected whether allele frequencies were pooled or stratified by ethnicity (Table 2).

\section{Prevalence of Subjective and Objective Cognitive Impairment}

A total of 193 participants completed FACT-Cog evaluation and 60 patients $(31.1 \%)$ reported subjective CRCI (Table 3 ). Among specific cognitive domain, the highest proportion of participants reporting cognitive decline was observed in the mental acuity $(28.5 \%)$ domains, followed by concentration (28.0\%), multi-tasking (25.9\%), verbal ability (20.2\%), functional interference (19.2\%), and memory (17.6\%).

A total of 120 participants completed CANTAB assessments. Participants who completed CANTAB assessments were younger, more likely to be pre-menopausal and attained higher education levels than those who did not. However, there was no difference in baseline anxiety and fatigue levels, proportion reporting subjective CRCI and $B D N F$ Val66Met genotypic distribution between the two groups of patients (Supplementary Table S1). A total of 59 individuals, representing nearly half of the patients $(49.2 \%)$ experienced decline in at least one cognitive domain (Table 3 ). The highest proportion of patients with cognitive decline was reported in the domain of sustained attention $(23.3 \%)$, followed by

Fig. 1 Study flow diagram

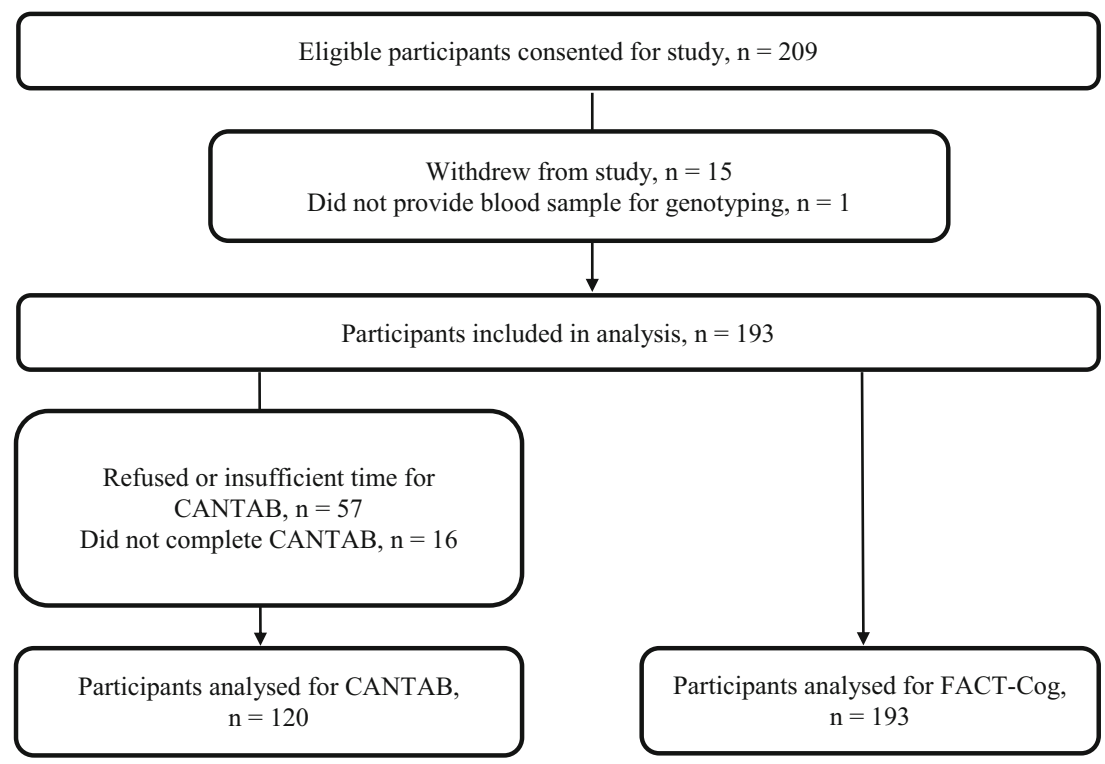


Table 1 Comparison of demographic and clinical characteristics of participants in the current cohort and original cohort

\begin{tabular}{|c|c|c|}
\hline & $\begin{array}{l}\text { Current cohort } \\
n=193\end{array}$ & $\begin{array}{l}\text { Original cohort } \\
n=145\end{array}$ \\
\hline \multicolumn{3}{|l|}{ Demographic characteristics } \\
\hline Age in years, mean (SD) & $51.9(8.9)$ & $50.8(8.8)$ \\
\hline \multicolumn{3}{|l|}{ Ethnicity, $n(\%)$} \\
\hline Chinese & $154(79.8)$ & $119(82.1)$ \\
\hline Malay & $19(9.8)$ & $15(10.3)$ \\
\hline Indian & $13(6.7)$ & $7(4.8)$ \\
\hline Others & $7(3.6)$ & $4(2.8)$ \\
\hline \multicolumn{3}{|l|}{ Education level, $n(\%)$} \\
\hline Primary school & $29(15.0)$ & $22(15.2)$ \\
\hline High school & $90(46.6)$ & $70(48.3)$ \\
\hline Pre-university & $35(18.1)$ & $29(20.0)$ \\
\hline Graduate/postgraduate & $38(19.7)$ & $24(16.6)$ \\
\hline Unknown & $1(0.5)$ & $0(0.0)$ \\
\hline \multicolumn{3}{|l|}{ Menopausal status, $n(\%)$} \\
\hline Premenopausal & $95(49.2)$ & $74(51.0)$ \\
\hline Postmenopausal & $98(50.8)$ & $71(49.0)$ \\
\hline \multicolumn{3}{|l|}{ Clinical characteristics } \\
\hline \multicolumn{3}{|l|}{ Cancer staging, $n(\%)$} \\
\hline Stage I & $27(14.0)$ & $32(22.1)$ \\
\hline Stage II & $127(65.8)$ & $71(49.7)$ \\
\hline Stage III & $39(20.2)$ & $41(28.3)$ \\
\hline Radiotherapy, $n(\%)$ & $128(66.3)$ & Not reported \\
\hline Surgery, $n(\%)$ & & Not reported \\
\hline Lumpectomy & $71(36.8)$ & \\
\hline Mastectomy & $122(63.2)$ & \\
\hline \multicolumn{3}{|l|}{ Chemotherapy, $n(\%)$} \\
\hline Anthracycline-based & $125(64.8)$ & $94(64.8)$ \\
\hline Non anthracycline-based & $68(35.2)$ & $51(35.2)$ \\
\hline \multicolumn{3}{|l|}{ Behavioral symptoms } \\
\hline Baseline fatigue, mean (SD) & $1.6(1.9)$ & $1.6(1.7)$ \\
\hline Baseline anxiety, mean (SD) & $6.9(7.2)$ & $6.7(6.1)$ \\
\hline Baseline insomnia, mean (SD) & $22.8(26.8)$ & $23.1(26.9)$ \\
\hline
\end{tabular}

response speed (14.2\%), working memory (14.2\%), learning and memory $(12.5 \%)$, and multitasking $(8.3 \%)$.

\section{Association of BDNF Genotypes with Cognitive Impairment}

After adjusting for potential confounders including anxiety and fatigue, Met allele carriers showed a consistent trend of decreasing odds of subjective CRCI across all domains; however, statistical significance was only observed in memory $(\mathrm{OR}=0.24,95 \% \mathrm{CI}=0.09-0.61)$; multitasking $(\mathrm{OR}=0.30$, $95 \% \mathrm{CI}=0.14-0.67)$; and mental acuity $(\mathrm{OR}=0.46,95 \%$ $\mathrm{CI}=0.21-0.99$ ). Apart for the mental acuity domain (unadjusted $\mathrm{OR}=0.53,95 \% \mathrm{CI}=0.27-1.04$ ), adjusting for potential confounders did not alter the significance of association (Table 4). In contrast, no significant associations were detected in all cognitive domains investigated for objective CRCI in both adjusted and unadjusted analysis (Table 4). Analysis performed assuming a general genetic model yielded results with similar trends (Supplementary Table S2).

\section{Trajectory of Fatigue and Anxiety and Association with BDNF Genotypes}

Mean scores of BFI and BAI, indicating fatigue and anxiety, respectively, showed an increasing trend over time (Table 5). Baseline fatigue and anxiety were also shown to be significant predictors of subjective CRCI in univariate analysis (Supplementary Table S3); however, further analysis showed that anxiety and fatigue levels over time were not associated with $B D N F$ Val66Met polymorphism.

\section{Meta-Analysis of Association between BDNF Genotypes and Subjective CRCI}

Meta-analysis of odds ratios from the original $(n=145)$ and current $(n=193)$ cohorts showed comparable trends with consistent directions of association. Significantly lower odds of CRCI were associated with Met allele carriers in the domains of memory $(\mathrm{OR}=0.34,95 \% \mathrm{CI}=0.17-0.70)$; multitasking $(\mathrm{OR}=0.33,95 \% \mathrm{CI}=0.18-0.59)$; and verbal ability (OR $=$ $0.46,95 \% \mathrm{CI}=0.24-0.88$ ) (Table 6 ). In addition, the pooled odds ratio of subjective CRCI based on total FACT-Cog score was also lower in Met allele carriers $(\mathrm{OR}=0.52,95 \% \mathrm{CI}=$ 0.29-0.94) (Table 6). No significant heterogeneity was detected between the two studies for all domains $\left(I^{2}=0-34 \%\right)$.

\section{Discussion}

Findings in this well-powered study and pooled results from both the original and current studies show that carriers of the $B D N F$ Met allele is associated with a trend towards lower odds of reporting self-perceived CRCI across different domains. This replicates the protective effect of $B D N F$ Val66Met on subjective CRCI we have observed in our previous work. Consistent with our previous report, $B D N F$ Val66Met was not associated with objective CRCI. Further meta-analysis of the original and the current cohort have also uncovered the protective effect between $B D N F$ Val66Met and global subjective CRCI. This is a novel finding that has not been reported in the literature.

To date, the only other studies investigating the effect of $B D N F$ Val66Met polymorphism on self-perceived cognitive function have been carried out in healthy individuals and did not report lower odds of subjective CRCI among BDNF Met carriers $[26,27]$. Therefore, we postulate that the protective 
Table 2 Genotype and allele frequencies of participants $(\mathrm{n}=193)$

\begin{tabular}{llllll}
\hline Genotype/allele & \multicolumn{2}{l}{ Ethnic subpopulation, $n(\%)$} & \multirow{2}{*}{ Pooled, $n(\%)$} \\
\cline { 2 - 5 } & Chinese & Malay & Indian & Others & \\
\hline $\begin{array}{l}\text { Total } \\
\text { Genotype }\end{array}$ & 154 & 19 & 13 & 7 & 193 \\
GG (Val/Val) & $35(22.7)$ & $10(52.6)$ & $4(30.8)$ & $3(42.9)$ & $52(26.9)$ \\
GA (Val/Met) & $84(54.6)$ & $6(31.6)$ & $7(53.8)$ & $4(57.1)$ & $101(52.3)$ \\
AA (Met/Met) & $35(22.7)$ & $3(15.8)$ & $2(15.4)$ & $0(0.0)$ & $40(20.7)$ \\
Allele & & & & & \\
G (Val) allele & $154(50.0)$ & $26(68.4)$ & $15(57.7)$ & $10(71.4)$ & $205(53.1)$ \\
A (Met) allele & $154(50.0)$ & $12(31.6)$ & $11(42.3)$ & $4(28.6)$ & $181(46.9)$ \\
$p$ value $^{\mathrm{a}}$ & 0.26 & 0.24 & 0.71 & 0.29 & 0.48 \\
\hline
\end{tabular}

${ }^{a} \mathrm{p}$ values of Chi-square tests to assess deviation from Hardy-Weinberg equilibrium

b "Others" include Sri Lankan, Filipino, and Burmese effect of $B D N F$ Val66Met polymorphism on cognitive impairment is conditional on the presence of active malignancy or ongoing cancer treatment, both which have been hypothesized as possible causes of CRCI [25]. This discrepancy may be explained by animal studies where the expression and release

Table 3 Proportion of participants with CRCI

\begin{tabular}{lc}
\hline & $\begin{array}{l}\text { Proportion of } \\
\text { participants, } n(\%)\end{array}$ \\
\hline Subjective CRCI $(n=193)$ & \\
Summation score & $60(31.1)$ \\
Cognitive domains & \\
Memory & $34(17.6)$ \\
Verbal ability & $39(20.2)$ \\
Concentration & $54(28.0)$ \\
Mental acuity & $55(28.5)$ \\
Functional interference & $37(19.2)$ \\
Multitasking & $50(25.9)$ \\
Decline in at least 1 domain & $88(45.6)$ \\
Objective CRCI $(n=120)$ & \\
Individual test measures & \\
RTI - Five choice reaction time & $17(14.2)$ \\
PAL - Total error (adjusted) & $15(12.5)$ \\
SWM - Between errors & $5(4.2)$ \\
SWM - Strategy & $15(12.5)$ \\
AST - Switching cost & $3(2.5)$ \\
AST - Congruency cost & $3(2.5)$ \\
AST - Reaction latency & $8(6.7)$ \\
RVP - A' & $8(6.8)$ \\
RVP - Latency & $20(16.7)$ \\
Cognitive domains & \\
Response speed & $17(14.2)$ \\
Learning and memory & $15(12.5)$ \\
Working memory & $17(14.2)$ \\
Multitasking & $10(8.3)$ \\
Sustained attention & $28(23.3)$ \\
Decline in at least 1 domain & $59(49.2)$ \\
Number of domains & \\
1 & $40(33.3)$ \\
2 & $11(9.2)$ \\
3 & $7(5.8)$ \\
4 & $1(0.83)$ \\
\hline & \\
& \\
& \\
&
\end{tabular}

of BDNF have been shown to be heavily dependent on other physiological elements, such as stress and inflammation, which are elevated in cancer patients undergoing treatment [28]. It has also been demonstrated that as cancer patients undergo chemotherapy, changes in plasma BDNF levels differ between $B D N F$ genotypes [29]. These observations indicate that the effect of genetic polymorphisms may be mediated by downstream mechanisms that vary in different disease states. To further elucidate the links between $B D N F$ Val66Met polymorphism and CRCI, it will be useful to investigate and compare differences in gene and protein expression between $B D N F$ genotypes in both healthy and cancer patient populations. This will not only enhance our understanding of how genetic factors influence the development of CRCI but also provide insights to the pathophysiology of CRCI.

Our earlier study showed a significant association of carrying the BDNF Met allele with decreased odds of selfperceived CRCI in the FACT-Cog domains of multitasking and verbal ability [7]. In this study, we were able to replicate our previous findings in multitasking ability but not in verbal ability although demographic and clinical characteristics of both the original and current cohorts were comparable. While similar directions of association were observed, the effect size of carrying the Met allele was smaller and did not achieve statistical significance (OR in this study $=0.57$, OR in original study $=0.34$ ). A possible explanation for this nonreplication could be genetic heterogeneity, where impairment in the verbal ability domain may not be specific to the $B D N F$ gene but also associated with other genes not covered in our studies. Another possibility is the phenomenon described as "winner's curse" where effect sizes are often found to be overestimated in initial genetic association studies [12, 30]. Replication attempts subsequently yield smaller effect sizes and as a result, studies are underpowered to detect a significant impact of genetic polymorphisms. In contrast, our metaanalysis has detected a significant association between the 
Table 4 Association of carrying $B D N F$ Met allele with CRCI

\begin{tabular}{|c|c|c|c|c|}
\hline \multirow[t]{2}{*}{ Variable } & \multicolumn{2}{|c|}{ Unadjusted analysis } & \multicolumn{2}{|l|}{ Adjusted analysis } \\
\hline & OR & $p$ value & OR & $p$ value \\
\hline \multicolumn{5}{|c|}{ Subjective cognitive impairment } \\
\hline & $(n=193)$ & & $(n=192)^{\mathrm{a}}$ & \\
\hline Total score & $0.63(0.32-1.24)$ & 0.18 & $0.62(0.29-1.30)$ & 0.21 \\
\hline Memory & $0.45(0.21-0.97)$ & $0.04^{\mathrm{b}}$ & $0.24(0.09-0.61)$ & $0.003^{\mathrm{b}}$ \\
\hline Multitasking & $0.43(0.22-0.86)$ & $0.02^{\mathrm{b}}$ & $0.30(0.14-0.67)$ & $0.003^{\mathrm{b}}$ \\
\hline Verbal ability & $0.58(0.28-1.24)$ & 0.16 & $0.57(0.24-1.38)$ & 0.22 \\
\hline Concentration & $0.98(0.48-1.99)$ & 0.96 & $0.86(0.38-1.90)$ & 0.70 \\
\hline Mental acuity & $0.53(0.27-1.04)$ & 0.07 & $0.46(0.21-0.99)$ & $0.047^{\mathrm{b}}$ \\
\hline Functional interference & $0.87(0.40-1.93)$ & 0.74 & $0.69(0.27-1.75)$ & 0.44 \\
\hline \multicolumn{5}{|c|}{ Objective cognitive impairment } \\
\hline & $(n=120)$ & & $(n=119)^{\mathrm{a}}$ & \\
\hline Response speed & $2.01(0.54-7.49)$ & 0.30 & $3.02(0.69-13.26)$ & 0.14 \\
\hline Learning and memory & $1.10(0.32-3.73)$ & 0.88 & $1.58(0.36-6.87)$ & 0.54 \\
\hline Working memory & $1.34(0.40-4.43)$ & 0.64 & $1.32(0.35-4.94)$ & 0.68 \\
\hline Multitasking & $0.36(0.10-1.33)$ & 0.12 & $0.32(0.06-1.70)$ & 0.18 \\
\hline Sustained attention & $2.12(0.73-6.13)$ & 0.17 & $3.02(0.88-10.35)$ & 0.08 \\
\hline
\end{tabular}

genetic polymorphism and global subjective CRCI, which was not reported in the previous study. The original cohort was not adequately powered to evaluate the effect size, and this limitation has been overcome by the combined analysis of both cohorts.

In contrast to subjective CRCI, we did not detect any significant association between $B D N F$ Val66Met polymorphism and objective CRCI, a trend which is consistent with findings from our previous work as well as other studies in breast and brain tumor patients [7, 31, 32]. The lack of agreement between trends and predictors of objective and subjective CRCI

Table 5 Association of $B D N F$ Met allele with fatigue and anxiety over time

\begin{tabular}{llll} 
Mean scores (SD) & & $\beta$ & $\beta$ value \\
\hline $\mathrm{T} 1$ & $\mathrm{~T} 2$ & $\mathrm{~T} 3$ &
\end{tabular}

Fatigue (BFI)

All participants 1.64 (1.89) 1.91 (2.03) 2.23 (2.05)

Met allele carrier

\begin{tabular}{llllll} 
No & $1.39(1.56)$ & $2.15(2.08)$ & $2.40(2.04)$ & Reference \\
Yes & $1.74(1.99)$ & $1.81(2.01)$ & $2.17(2.05)$ & -0.07 & 0.77 \\
Anxiety (BAI) & & & & & \\
All participants & $6.86(7.23)$ & $8.22(8.55)$ & $8.55(7.66)$ & & \\
$\begin{array}{l}\text { Met allele carrier } \\
\text { No }\end{array}$ & & & & \\
Yes & $6.00(8.79)$ & $8.87(8.52)$ & $9.40(7.42)$ & Reference \\
No & $6.82(6.60)$ & $7.98(8.58)$ & $8.23(7.74)$ & -0.75 & 0.48 \\
\hline
\end{tabular}

is counter-intuitive but has been commonly reported in literature [33]. Subjective reports of cognitive function are more reflective of the ability to complete daily activities, which require the coordination of different cognitive skills, some which may not have been measured by specific neuropsychological tests used to assess objective cognitive function. While these tests are widely acknowledged as the gold standard to assess cognitive function, the importance of subjective cognitive reports should not be dismissed as they portray the impact of impaired cognition on the daily functioning of patients. Future work in this area should emulate our study, incorporating both objective and subjective measures of cognitive function as both outcomes hold equal importance and are consistently shown to be poorly correlated with each other.

Past research has suggested that subjective cognitive impairment is closely linked to other chemotherapy-related symptoms such as anxiety, depression, and fatigue hence may be more indicative of emotional distress rather than compromised cognitive function [1]. One may therefore speculate that $B D N F$ Val66Met polymorphism may be protective against these accompanying symptoms rather than CRCI, explaining the lack of agreement between the association of $B D N F$ polymorphism with objective and subjective CRCI observed in our study. Nevertheless, anxiety and fatigue levels have been adjusted for in our analysis. Although we did not observe any significant associations in this study, other genetic association studies have also shown that unlike subjective $\mathrm{CRCI}$, anxiety, fatigue, and depression are not ameliorated but worsened among $B D N F$ Met carriers [34, 35]. 
Table 6 Pooled odds ratios of CRCI among patients carrying BDNF Met allele (Val/Met or Met/Met) compared to Val/Val genotype

\begin{tabular}{lllllll}
\hline Domain & Cohort & $\begin{array}{l}\text { OR } \\
(95 \% \mathrm{CI})\end{array}$ & & $\begin{array}{l}\text { Weight } \\
\text { Pooled OR } \\
\text { (95\% CI) }\end{array}$ & $p$ value & $I^{2}(\%)$ \\
\hline Summation & Previous & $0.40(0.16-1.04)$ & 39.1 & $0.52(0.29-0.94)$ & $0.03^{\mathrm{a}}$ & 0 \\
& Current & $0.62(0.29-1.30)$ & 60.9 & & & \\
Memory & Previous & $0.53(0.19-1.53)$ & 45.7 & $0.34(0.17-0.70)$ & $0.003^{\mathrm{a}}$ & 17 \\
& Current & $0.24(0.09-0.61)$ & 54.3 & & & \\
Multitasking & Previous & $0.37(0.15-0.91)$ & 43.0 & $0.33(0.18-0.59)$ & $<0.001^{\mathrm{a}}$ & 0 \\
& Current & $0.30(0.14-0.67)$ & 57.0 & & & \\
Verbal ability & Previous & $0.34(0.12-0.90)$ & 43.0 & $0.46(0.24-0.88)$ & $0.02^{\mathrm{a}}$ & 0 \\
& Current & $0.57(0.24-1.38)$ & 57.0 & & & \\
Concentration & Previous & $0.61(0.23-1.59)$ & 40.9 & $0.75(0.40-1.39)$ & 0.36 & 0 \\
\multirow{3}{*}{ Mental acuity } & Current & $0.86(0.38-1.90)$ & 59.1 & & & \\
& Previous & $1.03(0.37-2.86)$ & 36.5 & $0.62(0.33-1.15)$ & 0.13 & 34 \\
Functional interference & Current & $0.46(0.21-0.99)$ & 63.5 & & & \\
& Previous & $0.38(0.13-1.14)$ & 42.6 & $0.54(0.26-1.09)$ & 0.08 & 0 \\
& Current & $0.69(0.27-1.75)$ & 57.4 & & & \\
\hline
\end{tabular}

${ }^{\mathrm{a}} p<0.05$
Considering the combination of these facts, we are confident that our observations are due to true associations of $B D N F$ Val66Met polymorphism with reduced odds of subjective cognitive decline rather than the confounding effects of fatigue or other psychosocial factors.

In genetic association studies, replication attempts are crucial to confirm initial findings. Past studies have suggested several genetic polymorphisms as possible predictors of CRCI $[36,37]$ but to the best of our knowledge, none have never been successfully replicated. For example, the effect of $A P O E \& 4$ allele was first observed in breast cancer and lymphoma survivors, but similar associations have not been replicated in similar patient populations and cognitive domains $[32,38,39]$. The association of COMT Val158Met with cognitive impairment in breast cancer survivors has only been successfully replicated in patients with brain tumors [31]. Thus, this study is essential as it replicates observed associations of similar direction and strength in the same cognitive domains and study population.

A limitation of our study is that a different neuropsychological test battery, CANTAB, was employed to assess objective cognitive function as the Headminder system used in the original study was no longer commercially available. To reduce any potential discrepancy between Headminder and CANTAB, we ensured that all neuropsychological tests used were validated for similar cognitive domains. Furthermore, the RCI calculated to measure cognitive changes is standardized by dividing differences in test scores at two separate time points by the standard error of measurement. This ensures that score changes in both studies are comparable although different tests were utilized. Nevertheless, we acknowledge that the use of different cognitive assessment tools makes comparison between studies challenging. Furthermore, meta-analysis of findings on objective CRCI from both studies could not be performed as different neuropsychological tools were employed. In genetic association studies where replication attempts and meta-analyses are highly encouraged to increase the effective sample size for a more robust estimate of the genetic effect, it is imperative that similar tools to measure cognitive ability are used across different studies. It should also be noted that a proportion of participants did not complete CANTAB assessments. Given that patients who failed to complete CANTAB assessments were older, more likely to be post-menopausal, and received less education, the prevalence of objective cognitive decline may have been underestimated in this study. Nevertheless, we believe that this is unlikely to influence the lack of association that we observed between $B D N F$ genotype and objective CRCI, as age, education level, and menopausal status were controlled for in our regression analysis. Furthermore, the $B D N F$ Val66Met genotypic distribution between participants who had and had not completed CANTAB assessment were also found to be comparable.

In conclusion, carriers of the $B D N F$ Met allele were protected against global subjective CRCI, particularly in the domains of memory, multitasking, and verbal ability. Similar trends towards reduced odds of subjective CRCI in all other cognitive domains were also observed in both original and current cohorts. We have also confirmed that no association could be detected between $B D N F$ Val66Met polymorphism and objective cognitive function. As cancer- and treatmentrelated toxicities such as CRCI have been shown to have a devastating impact on cancer survivors, prediction models to estimate the risk of these toxicities should be established and tested for clinical use, so that survivors at risk can be targeted at an earlier stage for interventional measures to improve their daily functioning and quality of life. Genetic markers, such as $B D N F$ Val66Met, should be incorporated in these prediction models once they have been validated in other cancer 
populations. Augmented with more findings from gene and protein expression studies, this work will contribute to current knowledge on the biochemical pathways that are involved with the development of CRCI. This allows us to identify potential drug targets, which can be further screened for candidates of pharmacological interventions to attenuate the negative impact of CRCI among cancer patients.

Funding The authors acknowledge funding from the National Medical Research Council (NMRC/CIRG/1386/2014, NMRC/CIRG/1471/2017), National University of Singapore (R-148-000-233-114), and National Cancer Centre Singapore (NRFCB12131).

\section{Compliance with Ethical Standards}

Conflict of Interest The authors declare that they have no competing interests.

Open Access This article is distributed under the terms of the Creative Commons Attribution 4.0 International License (http:// creativecommons.org/licenses/by/4.0/), which permits unrestricted use, distribution, and reproduction in any medium, provided you give appropriate credit to the original author(s) and the source, provide a link to the Creative Commons license, and indicate if changes were made.

\section{References}

1. Ng T, Dorajoo SR, Cheung YT, Lam YC, Yeo HL, Shwe M, Gan YX, Foo KM et al (2018) Distinct and heterogeneous trajectories of self-perceived cognitive impairment among Asian breast cancer survivors. Psychooncology 27:1185-1192. https://doi.org/10. 1002/pon.4635

2. Wefel JS, Vardy J, Ahles T, Schagen SB (2011) International cognition and cancer task force recommendations to harmonise studies of cognitive function in patients with cancer. Lancet Oncol 12:703708. https://doi.org/10.1016/S1470-2045(10)70294-1

3. Cheung YT, Shwe M, Tan YP, Fan G, Ng R, Chan A (2012) Cognitive changes in multiethnic Asian breast cancer patients: a focus group study. Ann Oncol 23:2547-2552. https://doi.org/10. 1093/annonc/mds029

4. Cheung YT, Lim SR, Ho HK, Chan A (2013) Cytokines as mediators of chemotherapy-associated cognitive changes: current evidence, limitations and directions for future research. PLoS One 8: e81234. https://doi.org/10.1371/journal.pone.0081234

5. Cheung YT, Lee HH-L, Chan A (2013) Exploring clinical determinants and anxiety symptom domains among Asian breast cancer patients. Support Care Cancer 21:2185-2194. https://doi.org/10. 1007/s00520-013-1769-8

6. Ng T, Chan M, Khor CC, Ho HK, Chan A (2014) The genetic variants underlying breast cancer treatment-induced chronic and late toxicities: a systematic review. Cancer Treat Rev 40:11991214. https://doi.org/10.1016/j.ctrv.2014.10.001

7. Ng T, Teo SM, Yeo HL, Shwe M, Gan YX, Cheung YT, Foo KM, Cham MT et al (2016) Brain-derived neurotrophic factor genetic polymorphism (rs6265) is protective against chemotherapyassociated cognitive impairment in patients with early-stage breast cancer. Neuro-Oncology 18:244-251. https://doi.org/10.1093/ neuonc/nov162
8. Park H, Poo M (2013) Neurotrophin regulation of neural circuit development and function. Nat Rev Neurosci 14:7-23. https://doi. org/10.1038/nrn3379

9. Ahmed AO, Mantini AM, Fridberg DJ, Buckley PF (2015) Brainderived neurotrophic factor (BDNF) and neurocognitive deficits in people with schizophrenia: a meta-analysis. Psychiatry Res 226:113. https://doi.org/10.1016/j.psychres.2014.12.069

10. Altmann V, Schumacher-Schuh AF, Rieck M, Callegari-Jacques SM, Rieder CRM, Hutz MH (2016) Val66Met BDNF polymorphism is associated with Parkinson's disease cognitive impairment. Neurosci Lett 615:88-91. https://doi.org/10.1016/j.neulet.2016.01. 030

11. Toh YL, Ng T, Tan M, Tan A, Chan A (2018) Impact of brainderived neurotrophic factor genetic polymorphism on cognition: A systematic review. Brain Behav 8:e01009. https://doi.org/10. 1002/brb3.1009

12. Kraft P (2008) Curses - winner s and otherwise - in genetic epidemiology. Epidemiology 19:649-651. https://doi.org/10.1097/EDE. 0b013e318181b865

13. Wagner LI, Sweet J, Butt Z et al (2009) Measuring patient selfreported cognitive function: development of the functional assessment of cancer therapy - cognitive function instrument. J Support Oncol 7:W32-W39

14. Cheung YT, Lim SR, Shwe M, Tan YP, Chan A (2013) Psychometric properties and measurement equivalence of the English and Chinese versions of the functional assessment of cancer therapy-cognitive in Asian patients with breast cancer. Value Heal 16:1001-1013. https://doi.org/10.1016/j.jval.2013.06.017

15. Cheung YT, Foo YL, Shwe M, Tan YP, Fan G, Yong WS, Madhukumar P, Ooi WS et al (2014) Minimal clinically important difference (MCID) for the functional assessment of cancer therapy: Cognitive function (FACT-cog) in breast cancer patients. J Clin Epidemiol 67:811-820. https://doi.org/10.1016/j.jclinepi.2013.12. 011

16. Robbins TW, James M, Owen AM, Sahakian BJ, McInnes L, Rabbitt P (1994) Cambridge neuropsychological test automated battery (CANTAB): a factor analytic study of a large sample of normal elderly volunteers. Dement Geriatr Cogn Disord 5:266281. https://doi.org/10.1159/000106735

17. Owen AM, Downes JJ, Sahakian BJ, Polkey CE, Robbins TW (1990) Planning and spatial working memory following frontal lobe lesions in man. Neuropsychologia 28:1021-1034

18. Morris RG, Downes JJ, Sahakian BJ, Evenden JL, Heald A, Robbins TW (1988) Planning and spatial working memory in Parkinson's disease. J Neurol Neurosurg Psychiatry 51:757-766

19. Mendoza TR, Wang XS, Cleeland CS, Morrissey M, Johnson BA, Wendt JK, Huber SL (1999) The rapid assessment of fatigue severity in cancer patients: use of the brief fatigue inventory. Cancer 85 : 1186-1196

20. Ke Y, Ng T, Yeo HL, Shwe M, Gan YX, Chan A (2017) Psychometric properties and measurement equivalence of the English and Chinese versions of the Beck Anxiety Inventory in patients with breast cancer. Support Care Cancer 25:633-643. https://doi.org/10.1007/s00520-016-3452-3

21. Beck AT, Epstein N, Brown G, Steer RA (1988) An inventory for measuring clinical anxiety: psychometric properties. J Consult Clin Psychol 56:893-897

22. Luo N, Fones CSL, Lim SE, Xie F, Thumboo J, Li SC (2005) The European Organization for Research and Treatment of Cancer Quality of Life Questionnaire (EORTC QLQ-C30): validation of English version in Singapore. Qual Life Res 14:1181-1186. https:// doi.org/10.1007/s11136-004-4782-Z

23. Cheung Y-B, Thumboo J, Goh C, Khoo KS, Che W, Wee J (2004) The equivalence and difference between the English and Chinese versions of two major, cancer-specific, health-related quality-of-life 
questionnaires. Cancer 101:2874-2880. https://doi.org/10.1002/ cncr.20681

24. Ahles TA, Saykin AJ (2007) Candidate mechanisms for chemotherapy-induced cognitive changes. Nat Rev Cancer 7: 192-201. https://doi.org/10.1038/nrc2073

25. Moore HCF (2014) An overview of chemotherapy-related cognitive dysfunction, or "chemobrain". Oncology 28:797-804

26. Lim YY, Villemagne VL, Laws SM, Ames D, Pietrzak RH, Ellis KA, Harrington KD, Bourgeat $P$ et al (2013) BDNF Val66Met, A $\beta$ amyloid, and cognitive decline in preclinical Alzheimer's disease. Neurobiol Aging 34:2457-2464. https://doi.org/10.1016/j. neurobiolaging.2013.05.006

27. Kennedy KM, Reese ED, Horn MM, Sizemore AN, Unni AK, Meerbrey ME, Kalich AG Jr, Rodrigue KM (2015) BDNF val66met polymorphism affects aging of multiple types of memory. Brain Res 1612:104-117. https://doi.org/10.1016/j.brainres.2014. 09.044

28. Lubin FD, Roth TL, Sweatt JD (2008) Epigenetic regulation of bdnf gene transcription in the consolidation of fear memory. J Neurosci 28:10576-10586. https://doi.org/10.1523/JNEUROSCI. 1786-08.2008

29. Ng T, Lee YY, Chae J, Yeo AHL, Shwe M, Gan YX, Ng RCH, Chu PPY et al (2017) Evaluation of plasma brain-derived neurotrophic factor levels and self-perceived cognitive impairment post-chemotherapy: a longitudinal study. BMC Cancer 17:867. https://doi.org/ 10.1186/s12885-017-3861-9

30. Lohmueller KE, Pearce CL, Pike M, Lander ES, Hirschhorn JN (2003) Meta-analysis of genetic association studies supports a contribution of common variants to susceptibility to common disease. Nat Genet 33:177-182. https://doi.org/10.1038/ng1071

31. Correa DD, Satagopan J, Cheung K, Arora AK, Kryza-Lacombe M, Xu Y, Karimi S, Lyo J et al (2016) COMT, BDNF, and DTNBP1 polymorphisms and cognitive functions in patients with brain tumors. Neuro-Oncology 18:1425-1433. https://doi.org/10. 1093/neuonc/now057
32. Cheng H, Li W, Gan C et al (2016) The COMT (rs165599) gene polymorphism contributes to chemotherapy-induced cognitive impairment in breast cancer patients. Am J Transl Res 8:5087-5097

33. Hutchinson AD, Hosking JR, Kichenadasse G, Mattiske JK, Wilson C (2012) Objective and subjective cognitive impairment following chemotherapy for cancer: a systematic review. Cancer Treat Rev 38:926-934. https://doi.org/10.1016/j.ctrv.2012.05.002

34. Koh MJ, Jeung H-C, Namkoong K, Chung HC, Kang JI (2014) Influence of the BDNF Val66Met polymorphism on coping response to stress in patients with advanced gastric cancer. J Psychosom Res 77:76-80. https://doi.org/10.1016/j.jpsychores. 2014.04.008

35. Dooley LN, Ganz PA, Cole SW, Crespi CM, Bower JE (2016) Val66Met BDNF polymorphism as a vulnerability factor for inflammation-associated depressive symptoms in women with breast cancer. J Affect Disord 197:43-50. https://doi.org/10.1016/ j.jad.2016.02.059

36. Small BJ, Rawson KS, Walsh E, Jim HSL, Hughes TF, Iser L, Andrykowski MA et al (2011) Catechol-O-methyltransferase genotype modulates cancer treatment-related cognitive deficits in breast cancer survivors. Cancer 117:1369-1376. https://doi.org/10.1002/ cncr. 25685

37. Ahles TA, Saykin AJ, Noll WW, Furstenberg CT, Guerin S, Cole B, Mott LA (2003) The relationship of APOE genotype to neuropsychological performance in long-term cancer survivors treated with standard dose chemotherapy. Psychooncology 12:612-619. https:// doi.org/10.1002/pon.742

38. Vardy J, Dhillon HM, Pond GR, Rourke SB, Xu W, Dodd A, Renton C, Park A et al (2014) Cognitive function and fatigue after diagnosis of colorectal cancer. Ann Oncol 25:2404-2412. https:// doi.org/10.1093/annonc/mdu448

39. Koleck TA, Bender CM, Sereika SM, Ahrendt G, Jankowitz RC, McGuire KP, Ryan CM, Conley YP (2014) Apolipoprotein E genotype and cognitive function in postmenopausal women with early-stage breast Cancer. Oncol Nurs Forum 41:E313-E325. https://doi.org/10.1188/14.ONF.E313-E325 